\title{
Safety, Effectiveness and Tolerance of Buprenorphine- Naloxone in the Treatment of Opioid Dependence: Results from a Nationwide Non-Interventional Study in Routine Care
}

Authors

Affiliations
S. M. Apelt ${ }^{1,2}$, N. Scherbaum ${ }^{3}$, J. Gölz ${ }^{4}$, M. Backmund ${ }^{2,5}$, M. Soyka ${ }^{2,6}$

Affiliation addresses are listed at the end of the article

\author{
Key words \\ - buprenorphine-naloxone \\ - dependence treatment \\ - buprenorphine \\ naloxone \\ - opioids \\ routine care \\ safety
}

$\begin{array}{ll}\text { received } & 30.06 .2012 \\ \text { revised } & 26.10 .2012 \\ \text { accepted } & 29.10 .2012\end{array}$

Bibliography

DOI http://dx.doi.org/

10.1055/s-0032-1330033

Published online ahead of print:

4 January 2013

Pharmacopsychiatry 2013;

46: 94-107

(c) Georg Thieme Verlag KG

Stuttgart · New York

ISSN 0176-3679

\section{Correspondence}

S. M. Apelt, Dipl.-Psych.

Certum Consulting - Scientific

Research

Pfäffinger Straße 8

84564 Oberbergkirchen

Germany

Tel.: + 49/8637/7979913

Fax: +49/8637/797 9914

sma@certum-consulting.com

\section{Abstract}

$\nabla$

Introduction: Buprenorphine is well known in the treatment of opioid dependence. Despite a high safety profile and good tolerance buprenorphine has been subject to misuse and diversion. To reduce misuse the antagonist naloxone was added and the $4: 1$ combination of buprenorphine-naloxone was launched in Germany in March 2007. On the basis of the results from international clinical trials a non-interventional study was conducted to gather data on safety, effectiveness, retention and acceptability of buprenorphine-naloxone in the treatment of opioid dependent patients in routine care.

Methods: A nationwide multicentre 12-month prospective, non-interventional, post-marketing, surveillance study was carried out with 12 assessment points in $\mathrm{N}=384$ opioid dependent patients currently in maintenance treatment from $\mathrm{N}=69$ general practitioners, clinics and outpatient clinics in Germany.
Results: $\quad \mathrm{N}=337$ data sets were eligible for analysis. The rates of patients with serious and nonserious adverse events were low with $1.2 \%$ and $17.5 \%$, respectively. No deaths occurred during the observational period and only one hospitalization was documented. Concomitant drug use decreased for all illicit substances. Mental health and quality of life measured with standardized self-assessment questionnaires improved significantly. The 12 -month retention rate was $57.1 \%$. Of the $n=181$ patients still in treatment at the end of the observation period, $96.7 \%$ continued treatment with buprenorphine-naloxone.

Conclusion: The findings of the non-interventional study indicate high effectiveness and safety of buprenorphine-naloxone in the treatment of opioid dependence. The medication was well accepted by opioid dependent patients in long-term substitution treatment with substantial reductions of concomitant drug use and measurable improvement in quality of life.

\section{Introduction}

$\nabla$

Opioid dependence is a major health and social issue $[1,2]$ and is associated with an excess rate of somatic and psychiatric complications including HIV, hepatitis, depression, suicidality and antisocial behaviour $[1,3,4]$. Approximately 200000 persons in Germany have a risky use of illicit substances, excluding cannabis use [5]. Although the number of drug-related deaths continues to decrease still 1237 persons died in 2010 because of drug use, most of them because of heroin overdose (42.8\%), $12.5 \%$ of the deaths were related to methadone/levo-methadone alone or in combination with other drugs and $0.5 \%$ were related to buprenorphine alone or in combination with other drugs [5].
One of the reasons for the lowest number of drug-related deaths in the past 10 years [5] is opioid maintenance treatment which is an established and well-studied approach in opioid dependence and recommended by current treatment guidelines worldwide [6-9]. The main goals of opioid drug dependence treatment are risk and harm reduction, social reintegration, and interruption of the vicious circle of drug use and procurement crime. Furthermore the therapy aims to establish best possible conditions for the treatment of concomitant diseases [10]. Although abstinence is no longer the only primary goal, the long-term target of opioid drug dependence treatment is to support the patients to stop using drugs entirely [10].

In 2010 more than 77000 of approximately 200000 opioid-dependent patients in Germany 
were registered as currently in maintenance treatment with $\mathrm{d} / \mathrm{l}-$ methadone (58\%), levo-methadone (23\%), buprenorphine (19\%) and other substitution drugs including diamorphine $(0.3 \%)$ $[7,11]$. Both treatments with full opioid agonists (e.g., methadone) and partial agonist/antagonist (buprenorphine) have been found to be effective in reducing substance use and improving somatic, psychiatric as well as social functioning [2,12-14]. However the increasing level of diversion $[8,15,16]$ and the risk of fatal outcomes in opioid maintenance treatment have raised concerns about safety issues in the treatment of opioid dependence.

The combination of the partial mu-agonist/kappa-antagonist buprenorphine with the full mu-antagonist naloxone in a ratio of $4: 1$ was developed to improve treatment outcomes and to reduce the risk of diversion $[17,18]$. When the combination is administered sublingually as prescribed, naloxone is inactive because of its low sublingual bioavailability [19] and only the effects of buprenorphine are experienced $[16,20]$ blocking most of the mu-receptors [12]. But when the medication is administered parenterally (intravenous or nasal) the effects of naloxone are experienced for the first $15-90 \mathrm{~min}$ [21]. Both buprenorphine and naloxone have a very high bioavailability but naloxone binds more rapidly to the opioid mu-receptors than buprenorphine causing precipitated withdrawal if the user has full agonists in the body $[16,18]$. Thus the combination of buprenorphine with naloxone is expected to reduce the risk of intravenous or nasal misuse [19]. The combination minimizes the risk of opioid overdose and diversion by making it unattractive for selling $[17,21]$ because of the unpleasant experience directly after parenteral abuse $[18,21]$. In addition the potential pleasurable effects of buprenorphine are diminished due to the smaller and delayed agonist effects after the subsiding antagonistic effect of naloxone [21].

While a number of randomized clinical trials $[2,12,17,22]$ demonstrated the overall efficacy of buprenorphine-naloxone in the treatment of opioid dependence, to date no non-interventional observational studies on the effectiveness and safety of the novel buprenorphine-naloxone combination reflecting "real world" conditions with a profound and comprehensive assessment both for physicians and patients have been published. Such studies are essential to verify clinical trial results and to receive reliable safety data from routine care treatment. The study was designed to collect comprehensive safety and effectiveness data on a large patient sample in office-based routine opioid drug dependence treatment with buprenorphine-naloxone over a 12-month period (2008-2010).

\section{Methods}

$\nabla$

\section{Study goals}

The primary objectives of the non-interventional study was to describe the retention rate of patients pre-treated with buprenorphine, methadone, levo-methadone or another maintenance drug after 12 months of treatment with buprenorphinenaloxone under real-life conditions and to collect comprehensive safety data during switch to and treatment with buprenorphinenaloxone.

The secondary objectives were to describe the switch to the new medication in terms of dosing, mode of prescription and subjective effects. Data on effectiveness, acceptance and tolerance of

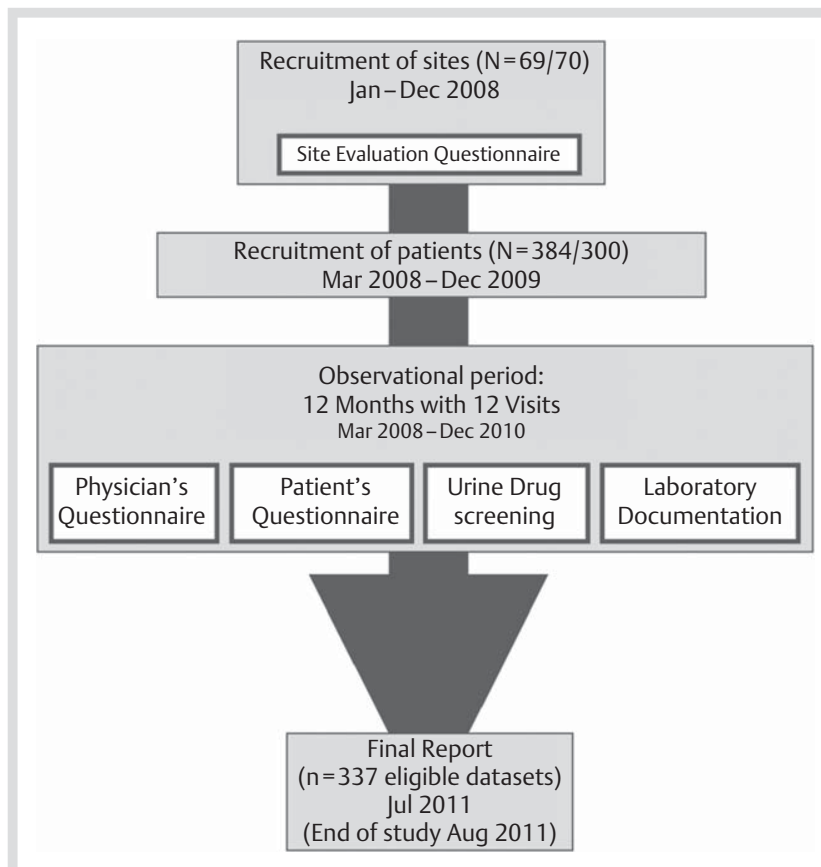

Fig. 1 Design of the non-interventional study with buprenorphinenaloxone.

opioid dependence treatment with buprenorphine-naloxone should be examined regarding met and unmet needs.

\section{Study design}

The study was a nationwide, prospective 12-month observational, non-interventional, post-authorization safety study (PASS) with patients currently in drug dependence treatment with another medication such as d/l-methadone, levo-methadone or buprenorphine for whom a switch to buprenorphinenaloxone was indicated and planned ( $\bullet$ Fig. 1). A comprehensive paper-based clinical research form was used for data capture. The study was part of the Risk Management Plan (RMP) for the newly marketed product buprenorphine-naloxone (Suboxone ${ }^{\circledR}$ ) and therefore a requirement of the European Medicine Agency (EMA). The study is registered with the National Institute of Health (NIH) at ClinicalTrials.gov (NCT00723749).

\section{Study population}

From $\mathrm{N}=69$ physicians working in addiction medicine and qualified pursuant to German Controlled Substances Regulation (Betäubungsmittelverordnung, BtMVV) § 5 (2) (1) (6) and with authorization granted by the Association of Statutory Health Insurance Physicians (Kassenärztliche Vereinigung, KV) $\mathrm{N}=384$ opioid-dependent patients were enrolled (total population). All patients over 15 years of age who had consented to opioid drug dependence treatment within the scope of medical, social and psychotherapeutic measures, for whom the switch to buprenorphine-naloxone was indicated and planned and who had signed the informed consent form could be included. The participating physicians were not subject to directives in terms of the use of buprenorphine-naloxone and prescribed the medication in the form of a conventional, commercially available product. Therapeutic indications and contraindications for opioid dependence treatment according to the Summary of Product Characterization (SmPC) for buprenorphine-naloxone and national treat- 


\begin{tabular}{|c|c|c|c|c|c|c|c|c|c|c|c|c|}
\hline Points of & Visit 1 & Visit 2 & Visit 3 & Visit 4 & Visit 5 & Visit 6 & Visit 7 & Visit 8 & Visit 9 & Visit 10 & Visit 11 & Visit 12 \\
\hline Method & Day 0 & Day 1 & Day 2 & Day 3 & Day 5 & Day 7 & Week 2 & Week 4 & Week 8 & Week 12 & Month 6 & $\begin{array}{l}\text { Month } 12 \text { or } \\
\text { Drop out documentation }\end{array}$ \\
\hline Patients informed consent (IC) & $x$ & & & & & & & & & & & \\
\hline Socio-demographics & $\mathrm{x}$ & & & & & & & & & & $\mathrm{x}$ & $\mathrm{x}$ \\
\hline Addiction/medical history & $\mathrm{x}$ & & & & & & & & & & & \\
\hline Drug, alcohol, tobacco use & $x$ & & & & & & & & & & $x$ & $x$ \\
\hline Vital parameters & $x$ & & & & & & & & & & $x$ & $x$ \\
\hline Physical examination & $\mathrm{x}$ & & & & & & & & & & $\mathrm{x}$ & $\mathrm{x}$ \\
\hline Co-morbidities & $\mathrm{x}$ & $\mathrm{x}$ & $\mathrm{x}$ & $\mathrm{x}$ & $\mathrm{x}$ & $\mathrm{x}$ & $\mathrm{x}$ & $\mathrm{x}$ & $\mathrm{x}$ & $\mathrm{x}$ & $\mathrm{x}$ & $x$ \\
\hline Co-medications & $\mathrm{x}$ & $\mathrm{x}$ & $x$ & $\mathrm{x}$ & $\mathrm{x}$ & $\mathrm{x}$ & $x$ & $\mathrm{x}$ & $\mathrm{x}$ & $x$ & $\mathrm{x}$ & $x$ \\
\hline Urine drug screening & $\mathrm{x}$ & & & & & & & $x$ & $x$ & $x$ & $x$ & $x$ \\
\hline Laboratory screening & $\mathrm{x}$ & & & & & & & & & $x$ & $x$ & $\mathrm{x}$ \\
\hline SF-36 Health Survey & $x$ & & & & & & & $x$ & & $\mathrm{x}$ & & $x$ \\
\hline oows/sows & $\mathrm{x}$ & $\mathrm{x}$ & $x$ & $\mathrm{x}$ & $x$ & $\mathrm{x}$ & $\mathrm{x}$ & $\mathrm{x}$ & $x$ & $x$ & $x$ & $\mathrm{x}$ \\
\hline VAS Craving & $x$ & $x$ & $x$ & $x$ & $x$ & $x$ & $x$ & $x$ & $x$ & $x$ & $x$ & $x$ \\
\hline SCL-90R & $x$ & & & & & & & $x$ & & $\mathrm{x}$ & $x$ & $x$ \\
\hline CGI/CGH-I & $x$ & $x$ & $x$ & $x$ & $x$ & $x$ & $x$ & $x$ & $x$ & $x$ & $x$ & $x$ \\
\hline AE/SAE & $x$ & $x$ & $x$ & $x$ & $x$ & $x$ & $x$ & $x$ & $x$ & $x$ & $x$ & $x$ \\
\hline Dosing, mode of allocation, cost unit & & $\mathrm{x}$ & $\mathrm{x}$ & $x$ & $\mathrm{x}$ & $x$ & $x$ & $x$ & $x$ & $x$ & $x$ & $\mathrm{x}$ \\
\hline Course of therapy & & $x$ & $\mathrm{x}$ & $\mathrm{x}$ & $\mathrm{x}$ & $x$ & $x$ & $x$ & $x$ & $x$ & $x$ & $\mathrm{x}$ \\
\hline \begin{tabular}{|l|} 
Reason for drop out \\
\end{tabular} & & & & & & & & & & & & $\mathrm{x}$ \\
\hline
\end{tabular}

Fig. 2 Flow chart parameters, methods and time points of observation. ment guidelines had to be observed when selecting patients for participation in the non-interventional study.

Of this total population $n=47$ datasets were excluded from the final analysis. Reasons were treatment not started (only baseline documentation available, $\mathrm{n}=18$ ), missing final documentation (month 12 or drop-out, $\mathrm{n}=21$ ) and incomplete documentation (no documentation of induction phase and follow-up documentation, $\mathrm{n}=8$ ).

The final analysis population of $n=337$ eligible datasets contains all patients with written informed consent, as approved by the ethics committee of the Ludwig-Maximilian University in Munich, as well as complete study documentation for at least baseline (day 0 ), start of treatment with buprenorphinenaloxone (day 1) and the final documentation either as end-ofobservation (month 12) or drop-out documentation. For $n=3$ patients day 1 documentation was missing and documentation of day 2 of treatment with buprenorphine-naloxone was used instead.

\section{Assessments}

Physicians questionnaire (third-party assessment)

The treating physicians informed eligible patients about the purpose of the study, the data collection procedure and the data privacy protection. Only after agreeing to all aspects and signing the informed consent form the baseline assessment, which was conducted before switching the patient to buprenorphinenaloxone, could be commenced.

The physician's questionnaire for evaluation of the patients was a paper-based assessment tool specially developed for the noninterventional study with 45 pages including 12 sections with several standardized instruments to document the following patient parameters: socio-demographics, substance use history, treatment history, co-morbidities, co-medication, concomitant drug use, urine drug screening, main reason for switch to buprenorphine-naloxone, treatment with buprenorphinenaloxone, premature discontinuation before end of observation, effectiveness measures with modified Clinical Global Impression (mCGI), Objective Opiate Withdrawal Scale (OOWS), and safety. It was the physician's decision which treatment data were transferred from the patient's medical chart to the questionnaire.

The CGI [23] is a standard measure for global assessments of illness consisting of 3 different global measures. In the study a modification of the Clinical Global Impression Severity scale
(CGI-S) and a modification of the Clinical Global Impression Improvement scale (CGI-I) was used. The OOWS [24] is a standardized scale for measuring the physically observable signs of opiate withdrawal for rating by the physician.

All adverse events (non-serious and serious including adverse drug reactions and pregnancies) were listed as they were spontaneously reported and documented at each visit by the treating physician.

\section{Patients questionnaires (self assessment)}

During the 12-month observational period all patients were asked to complete 4 standardized questionnaires in accordance with the schedule of observation points ( $\bullet$ Fig. 2): 1) Short Form 36 - Health Survey (SF-36) [25], a 36-item self-assessment questionnaire to survey the current health status with 2 modified indication specific questions in reference to drug dependence; 2) Subjective Opiate Withdrawal Scale (SOWS) [24], the subjective counterpart of the OOWS is a standardized scale for measuring the intensity of symptoms of opiate withdrawal from the perspective of the patient; 3) revised psychiatric Symptom Check-List (SCL-90R) [26,27], a standardized self-assessment tool to measure subjective impairment due to somatic and psychiatric symptoms; 4) visual analogue scale for craving (VAS Craving), an instrument specially invented for the non-interventional study by the first author containing twelve $100-\mathrm{mm}$ visual analogue scales for the substances alcohol, cannabis, amphetamines, hallucinogens, cocaine, barbiturates, benzodiazepines, opiates, d/l-methadone/levo-methadone, buprenorphine, codeine/DHC and other. Patients were asked to visualize their current craving for each of the listed substances.

\section{Assessment schedule}

To ensure eligible and valid data collection for comprehensive evaluation of induction and course of drug dependence treatment with buprenorphine-naloxone compared to baseline data before switch to the new medication, physician's and patient's questionnaires were scheduled for specific time points of observation (॰ Fig. 2).

\section{Statistics and analysis}

Except for socio-demographics, retention rate, regular end of treatment and safety all comparisons were made between baseline (day 0 ) and final assessment as regular end of observation (month 12) or premature discontinuation documentation (drop- 
out) for the total sample as well as for the analysis groups. Analyses concerning treatment with buprenorphine-naloxone used day $1 /$ start of treatment as baseline measures.

Single and multinomial logistic regression and chi-square tests were used for descriptive correlations between the defined analysis groups, start and end of observation. For numerical parameters, sample statistics, mean and standard deviation, minimum and maximum were calculated. For categorical data, absolute and relative frequencies were calculated. Data generated repeatedly in the course of time were evaluated per observation point. The differences between baseline and final assessment are shown for specific numerical data as absolute and relative difference.

Retention rates were estimated using Kaplan-Meier method and are presented as survival curves and 12-month survival estimates.

The options "not tested" and "no test" were set to missing values. For the option "no change" the status from the previous visit was carried forward.

Statistical significance was defined as p-values $<0.05$. Statistical analysis was done with STATA/SE 9 [28].

\section{Measures and specifications}

Retention rate: percentage of patients still in drug dependence treatment with buprenorphine-naloxone at the end of the observation period or who completed treatment after achieving a successful therapeutic outcome (regular end of treatment/ abstinence).

Safety: percentage of all documented non-serious and serious adverse events including adverse drug reactions, which were coded using MedDRA version 11.1 [29].

Effectiveness: improvement of scores from the standardized instruments mCGI for general health, SCL-90R for mental health, OOWS and SOWS for withdrawal; regular end of treatment (patient abstinent) documented by the treating physician in the final assessment as premature discontinuation documentation was defined as positive treatment outcome and patients were counted as completers.

Quality of life (QoL): improvement of scores from the standardized instrument SF-36 comparing baseline with the final assessment.

Acceptance and tolerance: reduction of concomitant drug use measured by urine drug screening, craving for illicit substances measured by the standardized instrument VAS craving and number of fresh needle marks.

\section{Analysis groups (post-hoc generation)}

Completers: patients still in drug dependence treatment with buprenorphine-naloxone at the end of observation (month 12) including patients with dropout reason regular end of treatment (patient abstinent from all illegal drugs including opiate-substitution).

Non-completers: patients with documented premature discontinuation of treatment with buprenorphine-naloxone for any reason other than regular end of treatment (patient abstinent).

Pre-treated: patients with documented current maintenance pharmacotherapy at baseline.
Untreated: patients without any documented previous maintenance pharmacotherapy at baseline [patients with no current maintenance treatment at study entry, but with a history of previous substitution treatment(s) are excluded from analysis between pre-treated and untreated patients].

Buprenorphine: patients in treatment with the mono compound buprenorphine at baseline.

(Levo-)methadone: patients in treatment with $\mathrm{d} / \mathrm{l}$-methadone or levo-methadone at baseline.

The term analysis groups refers to the above defined groups of completer/non-completer, pre-treated/untreated and buprenorphine/(levo-)methadone.

\section{Results}

$\nabla$

Data from $\mathrm{N}=337$ eligible patients was examined.

\section{Patient population}

Socio-demographics

- Table 1 summarizes patient characteristics at baseline for the total sample and all analysis groups. Most of the patients were male and in their mid-thirties, ranging from 18-62 years, and German nationality. Completers were older, married or living with a partner, working in a full-time job and living in their own flat. Higher rates of the more unfavourable characteristics such as unemployment, being divorced or single and being homeless are found in the group of non-completers.

\section{Addiction history}

As shown in 0 Table $1 \mathrm{~N}=244$ patients were in maintenance treatment with buprenorphine (66.4\%), d/l-methadone (20.9\%), levo-methadone $(9.8 \%)$ or another maintenance drug $(2.9 \%)$ at baseline. For $n=49$ patients the treatment with buprenorphinenaloxone was their first opioid drug dependence treatment and $\mathrm{n}=44$ patients were previously but not at baseline in maintenance treatment. Most of the participating patients had a long opioid addiction history from almost 14 years on average, ranging from 4 months to 50 years. Patients switched from the mono-compound buprenorphine and pre-treated patients had a significantly longer drug addiction history [patients with no current maintenance treatment at study entry, but with a history of previous substitution treatment(s) are excluded from analysis between pre-treated and untreated patients].

Almost all patients used opioids in their life (94.6\%) with no difference within the analysis groups. Non-completers revealed significantly higher rates in the use of benzodiazepines $(72.7 \%$ vs. $56.5 \%$ completer, $p=0.003)$, cocaine $(85.5 \%$ vs. $64.9 \%$, $\mathrm{p} \leq 0.001$ ), amphetamines ( $67.2 \%$ vs. $36.8 \%, \mathrm{p} \leq 0.001$ ), hallucinogens (42.5\% vs. $25.8 \%, p=0.002$ ), codeine (36.1\% vs. $18.8 \%$, $\mathrm{p}=0.001)$, barbiturates ( $29.0 \%$ vs. $11.2 \%, \mathrm{p} \leq 0.001)$. Pre-treated patients revealed significantly higher rates in the use of cocaine (75.4\% vs. 59.6\% untreated, $\mathrm{p}=0.039)$, benzodiazepines $(64.2 \%$ vs. $47.9 \%, p=0.035)$ and codeine $(27.4 \%$ vs. $10.9 \%, p=0.018)$. Significantly higher rates of life-time cannabis use were found in the group of patients switched from buprenorphine [91.9\% vs. $79.7 \%$ (levo-)methadone, $\mathrm{p}=0.008]$. 


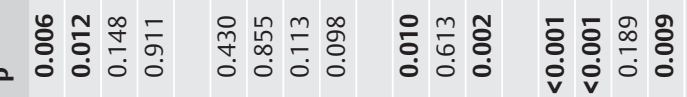

arge

s.

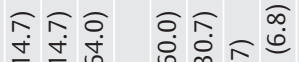

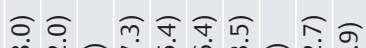

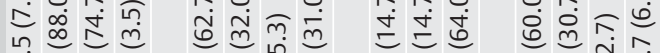

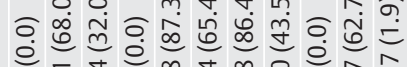

$m$ 范

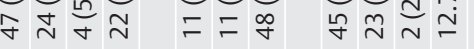

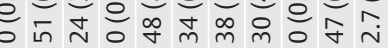

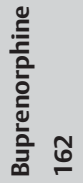

कำ

$\infty$

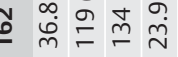

กิธ

m.

产

웅

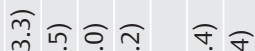



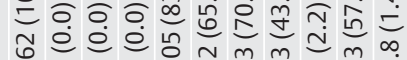

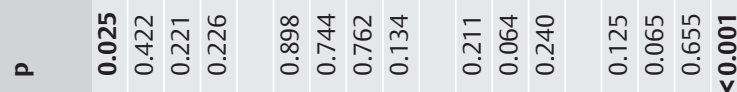

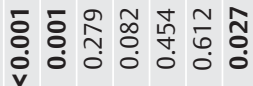

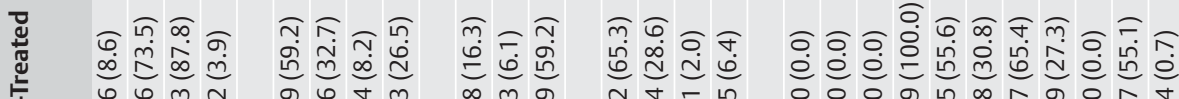

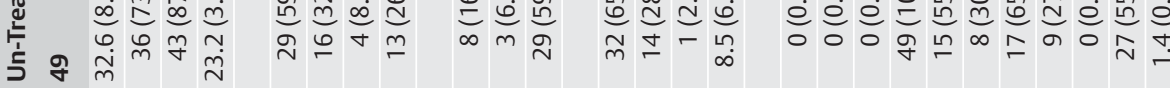

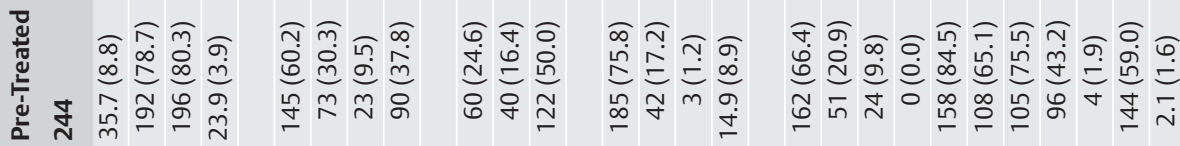

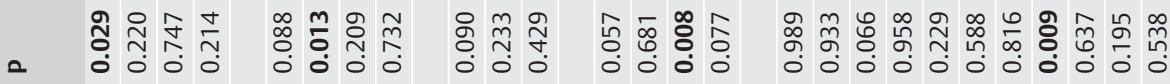



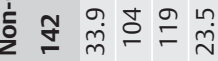

กู่

กิ

ธุ․․

$\hat{b} \underbrace{\circ}$

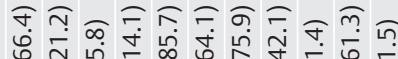

ñm

공

\& $\stackrel{\infty}{\sim} \stackrel{\infty}{\stackrel{\infty}{\sim}}$

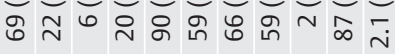

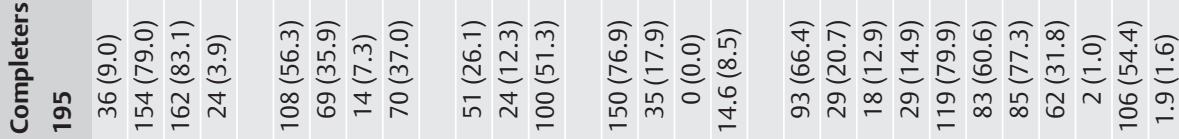

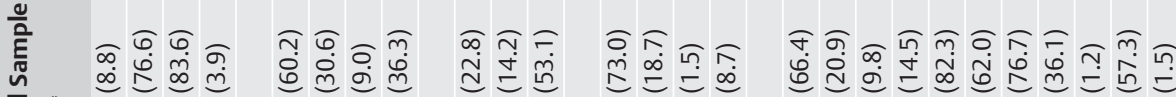

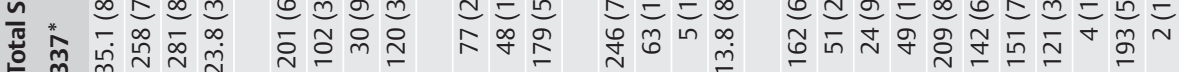

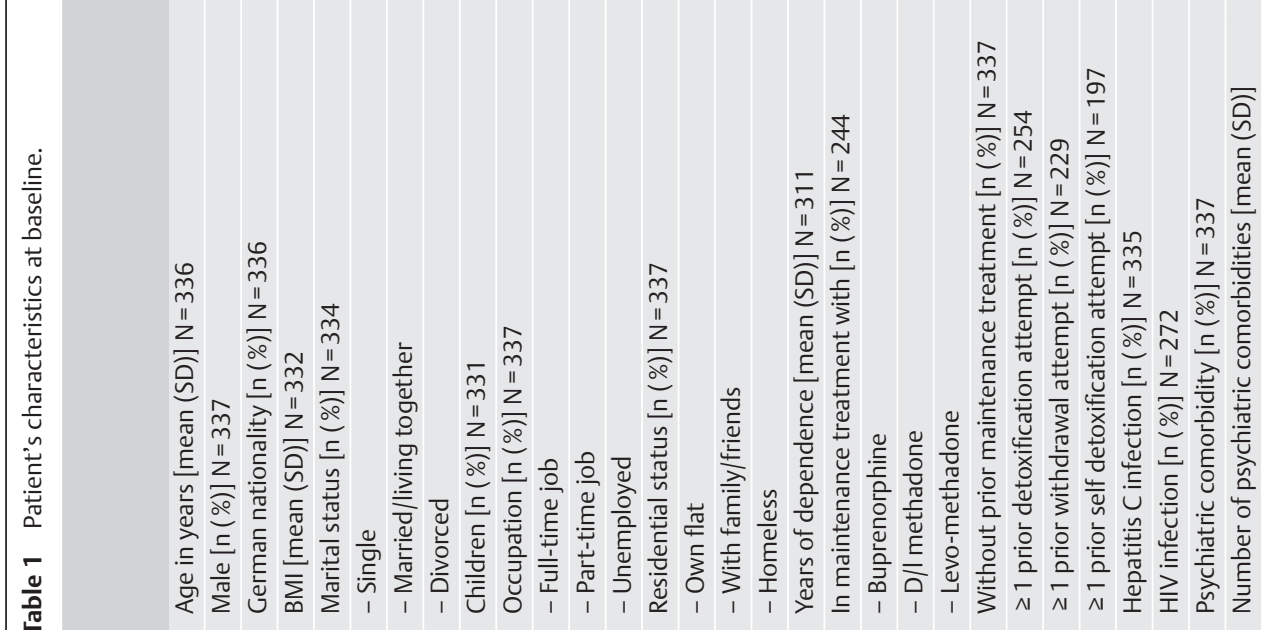


The average daily dosage for pre-treated d/l-methadone patients was $41.8 \pm 37.2 \mathrm{mg}(2-160 \mathrm{mg})$, levo-methadone patients $26.5 \pm 17.1 \mathrm{mg}(4-60 \mathrm{mg})$ and buprenorphine patients $7.7 \pm 4.3 \mathrm{mg}$ (1-24 $\mathrm{mg})$ at baseline.

\section{Retention rate and drop-out}

\section{Retention rate}

Of the total eligible patients $n=181$ were still in treatment at the end of observation after 12 months of treatment with buprenorphine-naloxone and $\mathrm{n}=14$ patients terminated their treatment during the observation period because they were rated as abstinent by their treating physician. The 12-month retention rate, analyzed with Kaplan-Meier estimator, was $57.1 \%$ for the total analysis population ( $\bullet$ Fig. 3 ). There were no differences between pre-treated and untreated patients ( $\Theta$ Fig. 3). A slightly higher retention rate was found in (levo-)methadone patients (० Fig. 4).

\section{Reasons for dropout}

$\mathrm{N}=142$ patient terminated treatment before end of observation. The most frequently documented reasons for drop out were "lost to follow up" (16.7\%), "concomitant drug use/relapse" (12.2\%), "side effects" (12.2\%) and "non-compliance/disciplinary reasons" (10.9\%). Significantly more untreated patients (16.7\% vs. $4.6 \%$ pretreated, $\mathrm{p}=0.033$ ) were rated as abstinent by the treating physician. No deaths occurred during the entire observational period. Only $\mathrm{n}=1$ hospitalization and $\mathrm{n}=3$ pregnancies led to premature termination of treatment with buprenorphine-naloxone.
Safety

Safety reporting for non-interventional studies is done according to regulations for routine care practice in Germany. The research forms of this study contained special sheets for documentation of all adverse events and the physician's folder provided reporting forms for serious adverse events but it was the physician's decision if an incident during the observation period required documentation and reporting, respectively. Therefore only non-serious and serious adverse events documented and reported by the treating physician could be included in the analysis. In this paper the adverse events reported were evaluated for the analysis population only.

\section{Serious adverse events (SAE)}

For $\mathrm{n}=4(1.2 \%)$ of the patients from the analysis population $(\mathrm{N}=337)$ there were $\mathrm{n}=4$ serious adverse events reported during the complete observational period including 30 days post-study time. The events, listed as system organ class and the term reported by the treating physician (in brackets) were $n=1$ psychiatric disorder (hospitalization because of suspected adjustment disorder), $\mathrm{n}=1$ social circumstances (concomitant drug use), $\mathrm{n}=1$ surgical and medical procedure (stay in hospital) and $\mathrm{n}=1$ nervous system disorder (epilepsy). One event was reported with certain correlation to the study drug (concomitant drug use), one with likely correlation (hospitalization because of suspected adjustment disorder), one with unlikely correlation (epilepsy) and one with unknown relation to the study drug (stay in hospital).

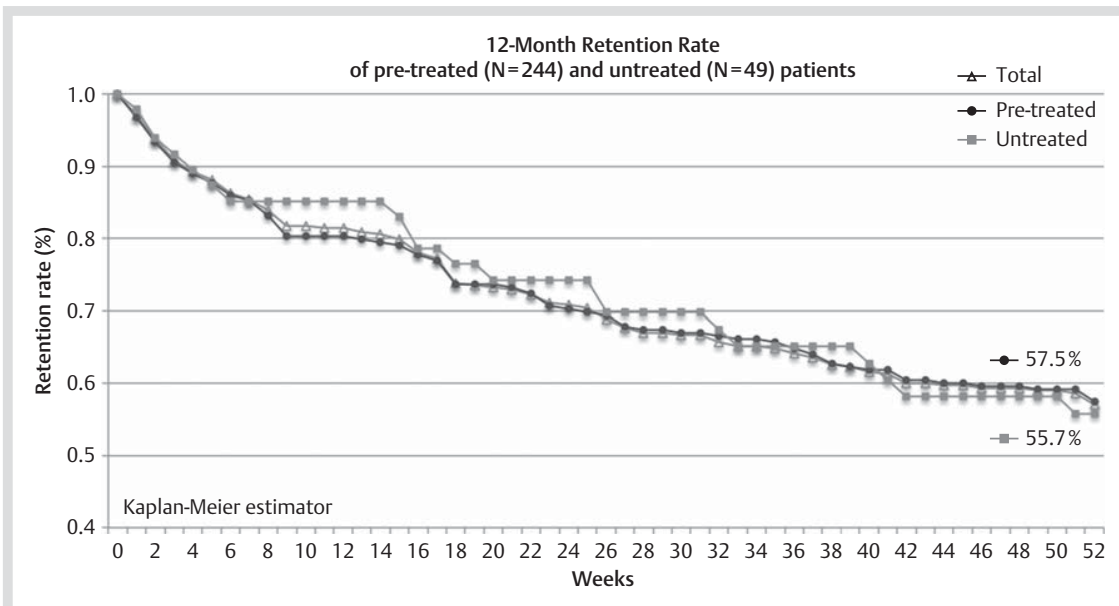

Fig. 3 12-month retention of the total analysis population $(N=337)$, pre-treated $(n=244)$ and untreated $(n=49)$ patients.

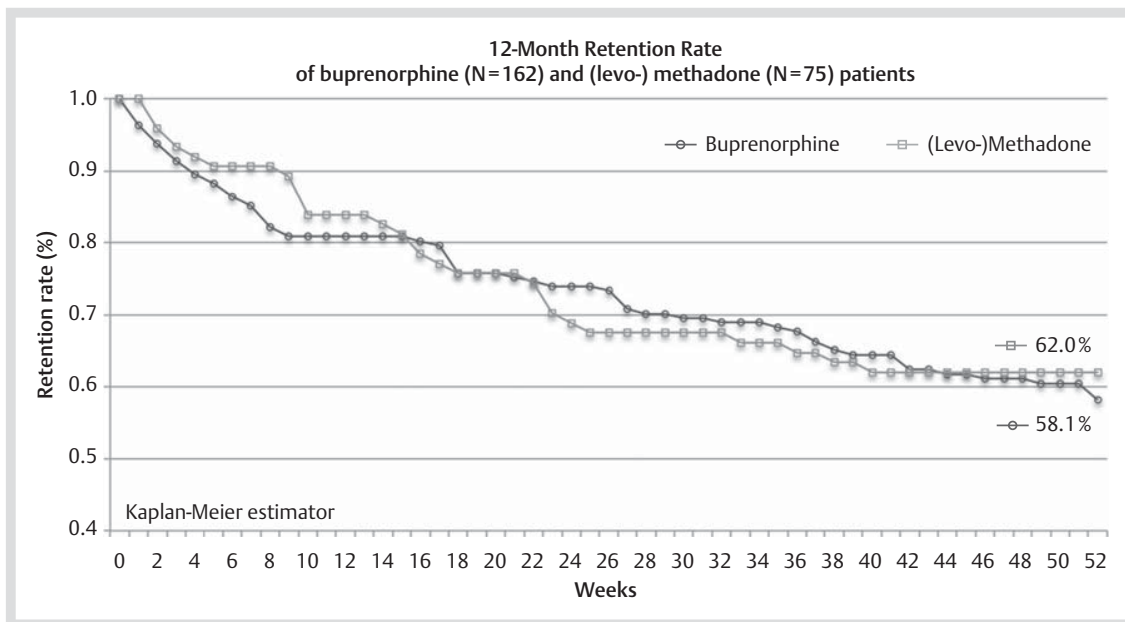

Fig. 4 12-month retention of buprenorphine $(n=162)$ and (levo-)methadone $(n=75)$ patients. 
No differences within the analysis groups were found in reference to the occurrence of SAEs. No deaths were reported during the study.

\section{Non-serious adverse events (NSAE)}

For $n=59(17.5 \%)$ patients $n=141$ non-serious adverse events were reported. NSAEs with a threshold of over $5.0 \%$ were psychiatric disorders (17.7\%), social circumstances (15.6\%, most of them concomitant drug use/non-compliance), gastrointestinal disorders (12.8\%), infections and infestations (12.8\%), nervous system disorders (9.9\%) and musculoskeletal and connective tissue disorders (9.2\%). 5 of the NSAEs were reported as certainly correlated to the study drug, $n=27$ likely related, $n=32$ possibly related, $n=67$ unlikely related and $n=10$ were reported as unknown concerning relation to the study drug. Significantly more non-completers $(23.2 \%$ vs. $13.3 \%$ completers, $\mathrm{p}=0.018$ ) and pre-treated patients ( $20.5 \%$ vs. $8.2 \%$ untreated, $\mathrm{p}=0.042$ ) were reported with non-serious adverse events. No difference was found between buprenorphine and (levo-) methadone patients concerning number of NSAEs.

\section{Treatment with buprenorphine-naloxone}

Reason for switch to buprenorphine-naloxone

The main reasons for switching to buprenorphine-naloxone were long-term maintenance treatment with or without abstinence as final goal (28.8\%), prior maintenance treatment not successful (21.4\%), planned detoxification treatment (17.8\%) and prevention of buprenorphine misuse (17.5\%). For $11.9 \%$ the physicians reported "patient's wish for take home" as reason for the switch to buprenorphine-naloxone.

\section{Dosing of buprenorphine-naloxone}

The mean induction dose of buprenorphine-naloxone was $9.2 \pm 5.1 \mathrm{mg}$ per day with a maximum of $32.0 \mathrm{mg}$. This dose slightly increased to $9.6 \mathrm{mg}$ on day 2 and 3 of the treatment with buprenorphine-naloxone and decreased continuously in the course of treatment to $7.7 \mathrm{mg}$ per day. Non-completers received generally higher doses, but those non-completers who were still in treatment at month $6(n=37)$ received virtually the same dose as completers ( $\bullet$ Fig. 5). Patients switched from d/l-methadone or levo-methadone received higher doses of buprenorphine-naloxone than patients switched from the mono-compound buprenorphine ( 0 Fig. 6). Doses of previous buprenorphine patients did not change during the course of treatment with buprenorphinenaloxone. Interestingly pre-treated and untreated patients' dose of buprenorphine-naloxone did not differ ( $\bullet$ Fig. 7).

Patients rated as abstinent during the observation period $(n=14)$ were not included in the analyses shown above. Their mean induction dose of buprenorphine-naloxone was $8.5 \pm 6.3 \mathrm{mg}$ which decreased rapidly to $7.0 \pm 4.7 \mathrm{mg}$ at day $7,4.6 \pm 2.6 \mathrm{mg}$ at week 4 and $2.0 \pm 1.6 \mathrm{mg}$ at their final assessment.

\section{Mode of prescription}

At the induction day most of the patients (87.1\%) received buprenorphine-naloxone on a daily basis at the practice of the treating physician and $8.4 \%$ as take-home prescription. All of the take-home prescriptions were documented for pre-treated patients and significantly more for buprenorphine patients (14.4\% vs. $5.3 \%$ (levo-)methadone, $\mathrm{p}=0.043$ ). Take-home prescription increased during treatment with buprenorphine-naloxone and was documented for $25.1 \%$ of the patients at the final assessment. Significantly more completers ( $30.1 \%$ vs. $18.3 \%$ non-completers, $\mathrm{p}=0.014$ ) received take-home at the time of their final assessment.

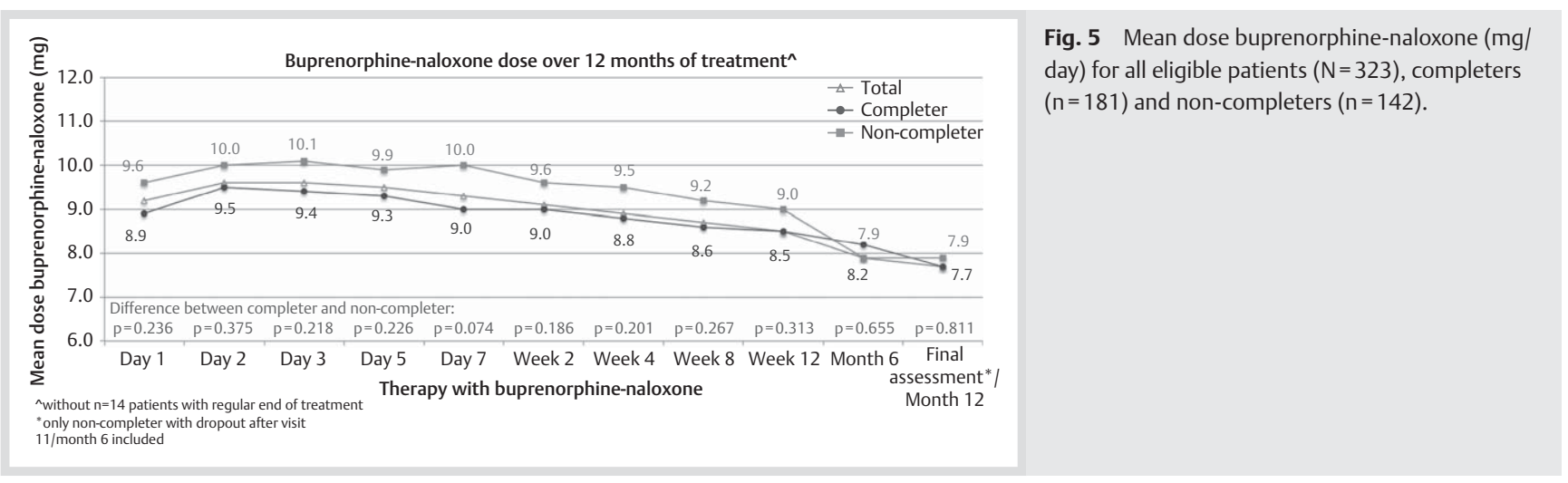

Fig. 5 Mean dose buprenorphine-naloxone (mg day) for all eligible patients $(\mathrm{N}=323)$, completers $(n=181)$ and non-completers $(n=142)$.

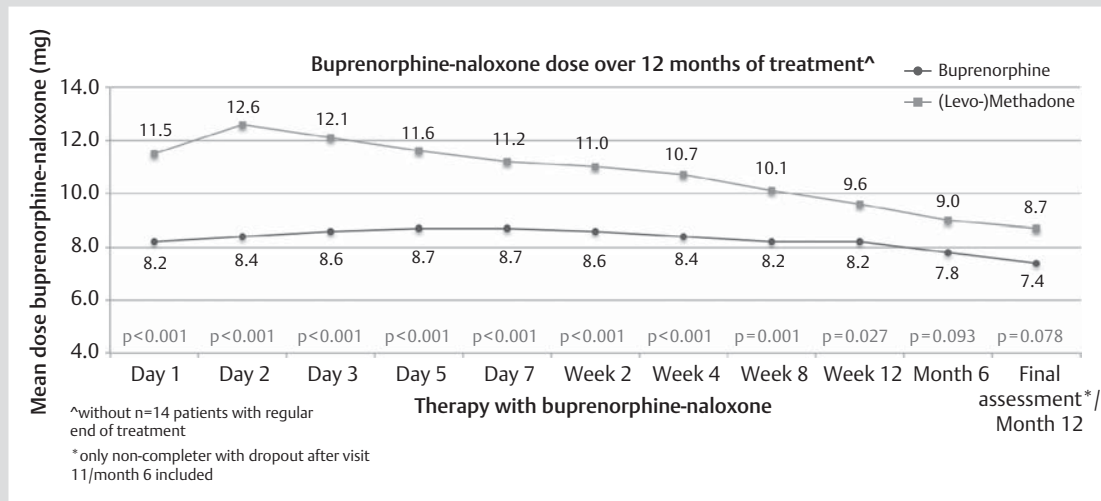

Fig. 6 Mean dose buprenorphine-naloxone (mg/ day) for buprenorphine patients $(n=159)$ and (levo-)methadone patients $(n=73)$. 


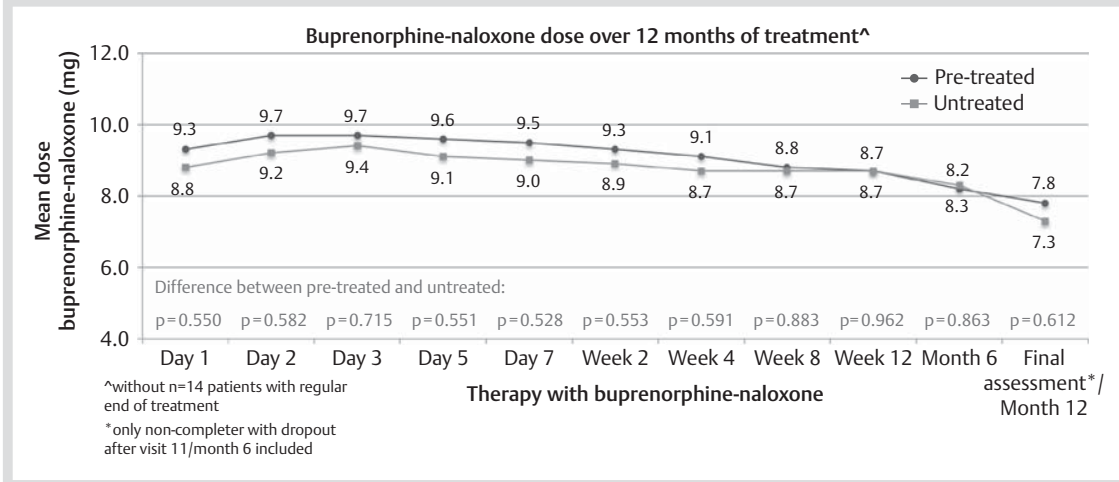

Fig. 7 Mean dose buprenorphine-naloxone (mg/day) for pre-treated $(n=239)$ and untreated $(n=45)$ patients.

\section{Quality of life}

\section{SF-36}

As shown in 0 Table 2 the scores of the standardized patient questionnaire SF-36 were relatively low at baseline but increased during treatment with buprenorphine-naloxone significantly for all scales. There was no difference between completers and non-completers at baseline except for pain. At the final assessment completers had significantly higher scores in all scales and non-completers revealed no substantial improvement from baseline to final assessment.

Pre-treated patients had higher scores at baseline but at the final assessment untreated patients achieved higher scores in all scales of the SF-36 and significantly for the scales emotional well-being and drug dependence compared to pre-treated patients.

At baseline buprenorphine patients achieved significantly higher rates compared to (levo-)methadone patients but no difference was found at the final assessment. While (levo-)methadone patients improved significantly in all scales, buprenorphine patients showed only for pain, social functioning, emotional role functioning and drug dependence significant improvement from baseline to final assessment.

\section{Effectiveness of the treatment with buprenorphine- naloxone \\ Mental health}

As shown in 0 Table 3 the mean scores of the SCL-90-R at baseline are higher in all scales for non-completers, untreated and (levo-)methadone patients. All patients achieved a significant improvement of psychiatric distress at their final assessment irrespective of analysis group.

\section{Modified Clinical Global Impression - Severity scale (mCGI-S)}

- Fig. 8 shows the modified CGI measuring the general health of the patient from the perspective of the physician. The categories were transformed to numeric scores $(0=$ very good to 5 = extremely bad). There was no difference between completers and non-completers but untreated and (levo-) methadone patients received significantly higher scores at baseline. According to the physicians the general health of all patients improved significantly during treatment with buprenorphine-naloxone except for non-completers. Their general health slightly worsened and the score was significantly higher $(p<0.001)$ at the final assessment compared to completers.

\section{Withdrawal}

Sows: The total score of the subjective opiate withdrawal scale at baseline was $17.2 \pm 13.5$ and decreased to $5.1 \pm 8.4$ at final assessment. Non-completers achieved a significantly higher score at baseline $(19.0 \pm 13.6$ vs. $15.9 \pm 13.4$ completers, $p=0.043$ ) and final assessment (11.7 \pm 11.7 vs. $3.9 \pm 7.0, \mathrm{p}<0.001)$. Untreated patients ( $20.8 \pm 14.7$ vs. $14.9 \pm 12.5$ pre-treated, $\mathrm{p}=0.005)$ and (levo-) methadone patients $(20.3 \pm 13.2$ vs. $11.8 \pm 10.5$ buprenorphine, $\mathrm{p}<0.001$ ) achieved a significantly higher score at baseline but did not differ from their comparison group at final assessment. All groups, except non-completer, achieved a significant reduction of subjective opiate withdrawal during the treatment with buprenorphine-naloxone.

oows: The total score of the objective opiate withdrawal scale reported by the treating physicians was $8.8 \pm 8.1$ at baseline and decreased significantly to $2.2 \pm 4.8(\mathrm{p}<0.001)$. From the physician's perspective there was no difference between completers and non-completers concerning opiate withdrawal at baseline, but at the final assessment non-completers received a significantly higher score ( $3.8 \pm 6.2$ vs. $1.1 \pm 3.0$ completers, $\mathrm{p}<0.001)$. Untreated ( $12.7 \pm 7.4$ vs. $7.2 \pm 7.6$ pre-treated, $\mathrm{p}<0.001)$ and (levo-) methadone $(11.1 \pm 7.7$ vs. $4.9 \pm 6.3$ buprenorphine, $\mathrm{p}<0.001)$ patients showed significantly more withdrawal symptoms at baseline. At the end of the observation physicians saw no difference between untreated and pre-treated patients, but in buprenorphine patients they identified more objective withdrawal symptoms $[2.2 \pm 4.4$ vs. $1.1 \pm 3.3$ (levo-)methadone, $\mathrm{p}=0.050]$.

\section{Regular end of treatment}

For $n=14$ patients the premature discontinuation within the 12-month observation period was the regular end of treatment with buprenorphine-naloxone because they were rated as abstinent by their treating physician $(4.2 \%$ of the total eligible patient population). Significantly more patients without prior maintenance treatment became abstinent ( $8.2 \%$ vs. $2.1 \%$ pre-treated, $\mathrm{p}=0.002$ ). No difference was found between patients with prior buprenorphine treatment and treatment with (levo-)methadone.

\section{Acceptance and tolerance \\ Concomitant drug use}

According to the results of urine drug screenings ( $\bullet$ Table 4) approximately one-third of the patients had a current use of opioids and cannabis at baseline. Significantly higher rates of 


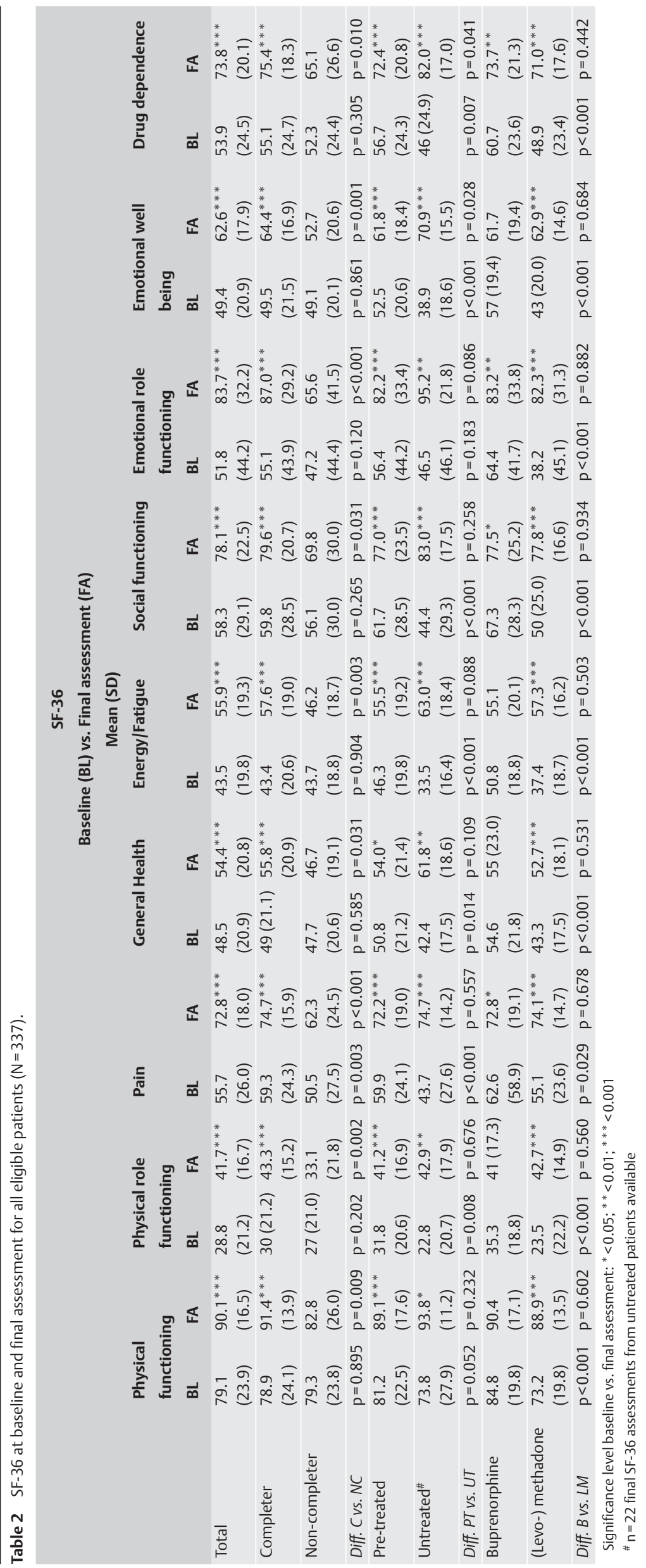




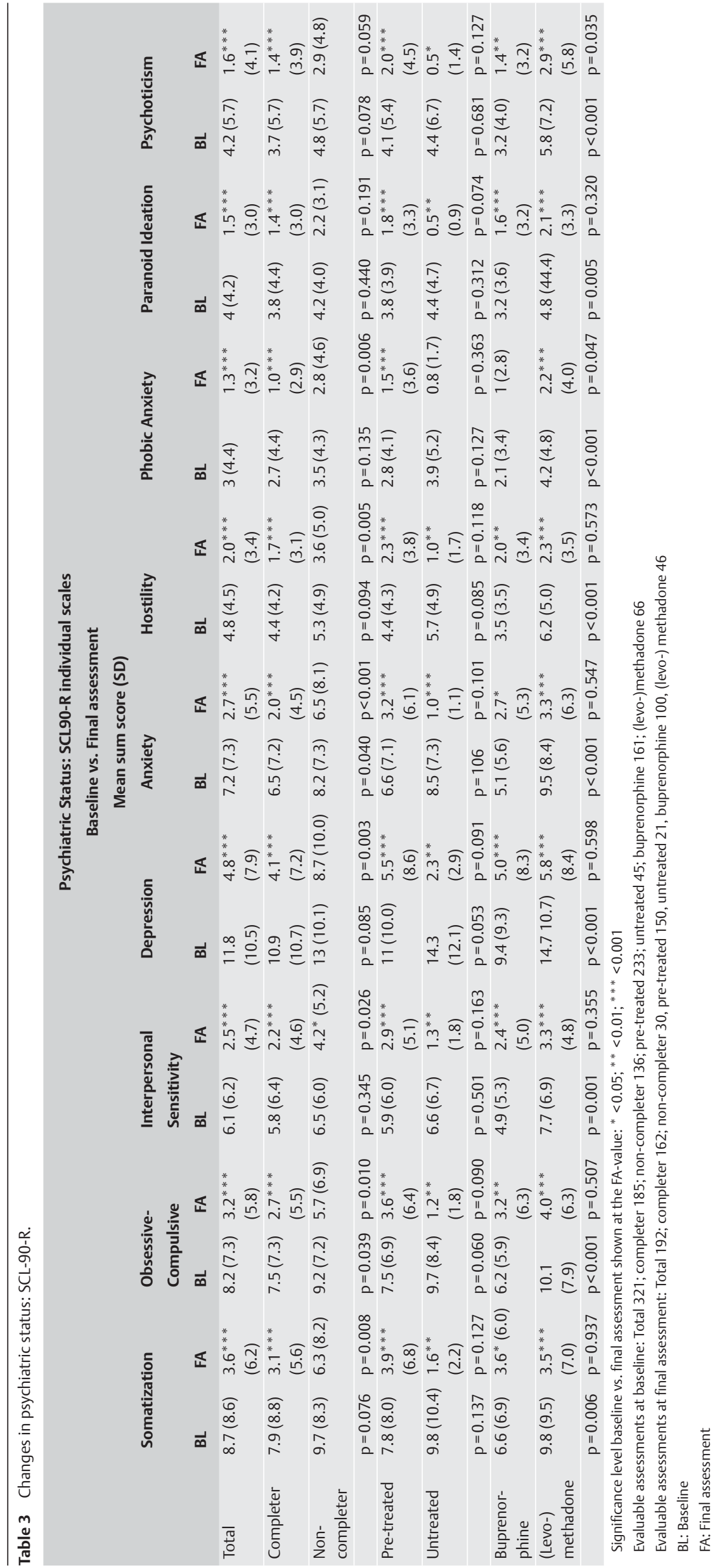




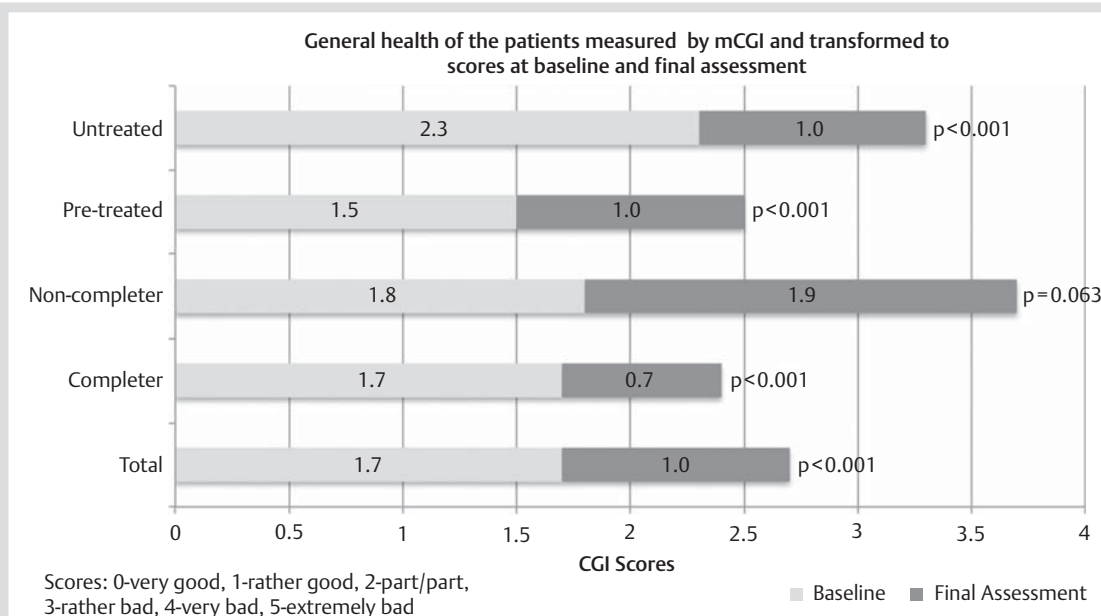

Fig. 8 Modified CGI score at baseline and final assessment for all eligible patients $(\mathrm{N}=337)$.

3-rather bad, 4-very bad, 5-extremely bad

opioid use were found in non-completers and untreated patients. Significantly more non-completers used cocaine and benzodiazepines, significantly more untreated patients used benzodiazepines and amphetamines and significantly more (levo-) methadone patients used cannabis. During the treatment with buprenorphine-naloxone urine drug screenings revealed a significant reduction of drug use for all illicit substances except barbiturates. Significantly higher rates of opioid use, cocaine, benzodiazepines and amphetamines were found in non-completers compared to completers. Significantly more (levo-)methadone patients were found to be active cannabis users at the final assessment.

\section{Opiate craving (VAS)}

Patients reported the highest craving at baseline and the highest decrease of craving during treatment with buprenorphinenaloxone for opiates $(32.3 \pm 33.2$ vs. $7.2 \pm 17.3$, $p<0.001)$. Noncompleters ( $38.9 \pm 35.4$ vs. $27.6 \pm 30.9$ completers, $\mathrm{p}=0.003$ ) and untreated patients $(46.4 \pm 37.9$ vs. $25.7 \pm 29.7$ pre-treated, $\mathrm{p}<0.001$ ) reported a significantly higher total score at baseline, but only non-completers reported a relatively high craving score at the final assessment $(24.7 \pm 32.5$ vs. $4.2 \pm 10.5$ completers, $\mathrm{p}<0.001)$. At the end of the observation untreated patients did not differ from pre-treated patients concerning craving for opiates. There was no difference found between buprenorphine and (levo-)methadone patients at baseline and final assessment. Nevertheless the reduction of craving for opiates was significant for all groups including non-completer.

\section{Fresh needle marks}

Physicians documented fresh needle marks for $13.5 \%$ at baseline, for slightly more non-completers (17.3\% vs. $10.8 \%$ completers, $\mathrm{p}=0.086)$ and significantly more untreated patients $(20.4 \%$ vs. $9.5 \%$ pre-treated, $p=0.029$ ). Most of these patients had a positive urine drug screening for opioids (75.6\%).

At the final assessment physicians reported fresh needle marks for $n=10$ patients, all of them were non-completers, $n=4$ were pre-treated, $n=2$ untreated and $n=4$ were switched from buprenorphine. Most of these patients had positive drug screenings for opioids $(n=7)$. Physicians reported no fresh needle marks for (levo-)methadone patients.

\section{Discussion}

The results of this non-interventional study underline the overall effectiveness of opioid drug dependence treatment with the 4:1 combination buprenorphine-naloxone. In line with findings in a previous naturalistic study in routine care [30] the 12-month retention rate of patients induced or switched to buprenorphine-naloxone was 55.7-62.0\% depending on previous maintenance treatment. These rates are also in line with results on retention of patients receiving standard methadone treatment [30-32]. No deaths occurred and the very low rate of adverse events emphasizes the high safety profile of buprenorphinenaloxone. Significant improvements in almost all evaluated domains during the 12-month observation period, irrespective of study completion and previous maintenance treatment, verify the effectiveness of the medication found in previous clinical trials $[2,12,17,19,22]$. As reported by Wittchen et al. 2008 [30] in their naturalistic study in 2694 patients, the same proportion of patients $(4.2 \%)$ had achieved abstinence during the observational period. Since a certain period (e.g., 5 years) of abstinence is required to reduce the risk of future relapse [3] we recommend a follow-up study to verify the status of patients with documented regular end of treatment because of abstinence. Compared to international findings on dosing of buprenorphinenaloxone between $16-24 \mathrm{mg} /$ day [33], patients observed in this non-interventional study received lower doses of $10 \mathrm{mg} /$ day on average, which decreased to an average of $8 \mathrm{mg} /$ day at the end of the 12-month observation, irrespective of study completion and previous maintenance treatment. Patients switched from $\mathrm{d} / \mathrm{l}$ methadone or l-methadone received significantly higher doses of $11 \mathrm{mg} /$ day on average decreasing until end of study to slightly but non-significantly higher doses of approximately $9 \mathrm{mg} / \mathrm{day}$. Dosing is a critical aspect in the treatment and retention of opioid dependent patients - it is important to alleviate the patient's cravings and withdrawal symptoms. The data of this non-interventional study reveal a significant relation between study completion status and withdrawal symptoms as well as opioid craving scores. Non-completers started with significantly higher subjective opiate withdrawal symptoms and craving which was still significantly higher at the time of their premature discontinuation of treatment with buprenorphinenaloxone. According to the physicians there was no difference concerning objective opiate withdrawal symptoms between non-completers and completers at baseline, but at the final 

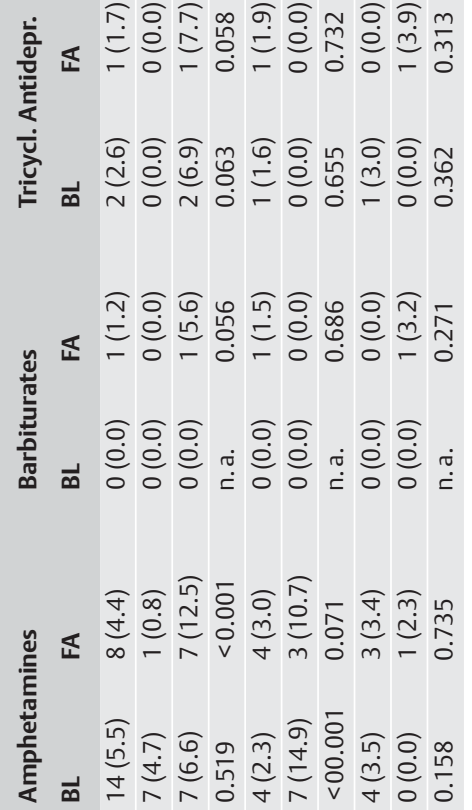

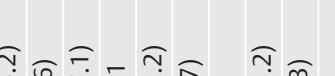
$\bar{o}$ 气

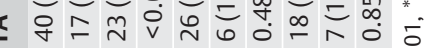



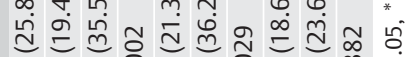

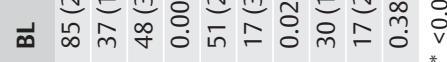

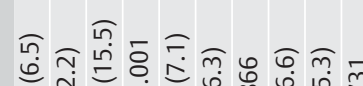

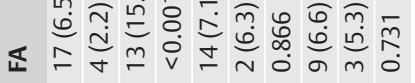

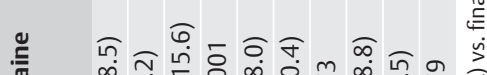

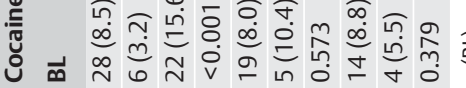

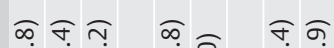

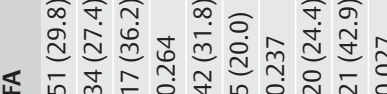



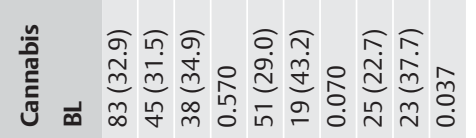

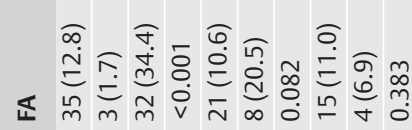

匹

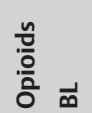

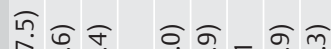

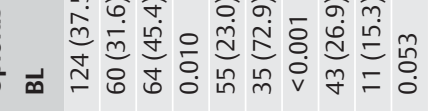
容 $\frac{20}{2}$ 隹 assessment they reported significantly higher objective withdrawal symptoms for non-completers. Completers achieved a significant reduction of subjective opiate withdrawal during the treatment with buprenorphine-naloxone, however the reduction of craving for opiates was significant for all groups including non-completers compared to baseline.

Take-home prescription is an important factor for reintegration into social and occupational life, because it enables the patient to start or stay in employment due to more flexibility in daily routine. $11.9 \%$ of all eligible patients wanted to switch to buprenorphine-naloxone for take-home prescription. At start of treatment with buprenorphine-naloxone a minority of patients received take-home prescription (8.4\%) and all of these were pre-treated patients. At the end of the observation $25.1 \%$ of all observed patients received take-home prescription. The decision for a take-home prescription is discretionary to the treating physician in compliance with $\S 5$ (8) BtMVV (Controlled Substances Prescription Regulation). The patient may receive a takehome prescription for up to 7 consecutive days if the patient is in stable maintenance treatment, without relevant concomitant drug use and use of other substances that interact with the maintenance drug and therefore endanger his health [34].

Psychiatric comorbidities are very common in this patient population [35]. At baseline the scores of the SCL-90-R were relatively low with higher rates in all scales for non-completers, untreated patients and (levo-)methadone patients. Apart from non-completers all eligible patients achieved a significant reduction of psychiatric distress at their final assessment. However non-completers did reach lower scores (except for the scale interpersonal sensitivity) at their final assessment. These results are in line with the findings shown by Lieb et al. 2010 [35]. Opioid dependent patients with high scores in psychiatric distress should receive specific care with integrated treatment for both opioid dependence and psychiatric disorder.

The scores of the standardized patient questionnaire SF-36 measuring the quality of life in terms of general health, emotional and social functioning were relatively low at baseline but increased significantly during treatment with buprenorphinenaloxone for all scales. At baseline no difference between completers and non-completers was found, but at the final assessment completers had significantly higher scores and non-completers revealed no substantial improvement during treatment. These findings suggest that the treatment with buprenorphinenaloxone improves the patient's perception of his emotional and social condition and his ability to reintegrate into a functioning social life. Since non-completers obviously did not benefit in this domain there might be other influencing factors, such as dosing, withdrawal and psychiatric comorbidity that need to be explored in order to support special patient groups in the treatment with buprenorphine-naloxone at an early stage.

The results are in line with an Italian longitudinal outpatient survey, which compared the treatment of opioid dependence with buprenorphine-naloxone and methadone [32]. The retention rate was similar in both groups but significant improvements of social life, educational level and concomitant drug use were found in patients treated with buprenorphine-naloxone.

The non-interventional study with buprenorphine-naloxone provides a unique database with comprehensive, reliable and valid data on opioid drug dependence treatment with buprenorphine-naloxone in routine care. However the major limitation is the strict observational nature of the study and the lack of a control group. Confounding factors, which may occur during a non- 
interventional study, cannot be controlled in contrast to clinical trials with exact regulations and complete treatment protocol. Thus these uncontrolled confounding factors may influence treatment outcomes. All measures used in this paper were of descriptive quality; effects and correlations need to be interpreted with caution. Since this observational study was part of the Risk-Management-Plan and based on a commitment to the European Medicine Agency (EMA) after market authorization of the product buprenorphine-naloxone, no control group was planned and necessary. Nevertheless this database with its broad range of variables, standardized assessments and parameters describing the course and outcome of the treatment with buprenorphine-naloxone from the physician's and the patient's perspective allows detailed analyses on safety, somatic and psychiatric health as well as subjective and objective effects in reference to special patient groups at different time points. This is the main advantage of this non-interventional study in routine care. In summary, the results indicate high acceptance and tolerance of the treatment accompanied by significant improvements in psychiatric, somatic and social functioning. According to these data buprenorphine-naloxone has an excellent safety profile also in comparison to methadone with a low risk especially for serious intoxications [9]. There are increasing safety concerns for intoxications with opioid prescription drugs, with no corresponding European data. Data from surveillance studies like this may help to better estimate the safety risk associated with the use of opioid maintenance treatment.

Although only pre-treated patients were the target study population some physicians included a small group of untreated patients and they provided encouraging results. The treatment with buprenorphine-naloxone was highly successful for patients without any experience in maintenance treatment with direct transfer from street heroin use to buprenorphine-naloxone treatment.

The characteristics of non-completers need to be analysed further to identify those at risk for negative outcome. Analyses should focus on identifying ways to retain such patients in treatment and heighten their chances for treatment success.

\section{Acknowledgements \\ $\nabla$}

None.

\section{Funding Source, Contribution and Conflict of Interest $\nabla$}

Essex Pharma GmbH \& Reckitt Benckiser Pharmaceuticals conducted this strictly observational, non-interventional study. All physicians received honoraria for the additional effort to document the start and course of the treatment with buprenorphinenaloxone in the comprehensive paper-based clinical research form. The honoraria were in line with the German medical fee schedule (Gebührenordnung für Ärzte, GOÄ) which controls the invoicing of medical attendance outside of the statutory health insurance. All authors contributed to and have approved the final manuscript.

Sabine M. Apelt has a financial interest/arrangement or affiliation with one or more organizations that could be perceived as a real or apparent conflict of interest in the context of the subject of this presentation. She receives or has in the past 3 years received honoraria from: Schering Plough, Essex Pharma GmbH,
MSD SHARP \& DOHME GmbH and Reckitt Benckiser Pharmaceuticals. In the past 3 years Norbert Scherbaum received honoraria from Essex Pharma $\mathrm{GmbH}$, Reckitt Benckiser, Molteni and Sanofi-Aventis. Jörg Gölz has no conflict of interest. Markus Backmund has no conflict of interest. For the past 5 years Michael Soyka has received travel grants, unrestricted research funding or has worked as a consultant for Presspharm, Reckitt Benckiser, Lilly, Astra Zeneca, Roche, Essex Pharma GmbH, Lundberg and Sanofi Aventis.

\section{Affiliations}

${ }^{1}$ Certum Consulting - Scientific Research, Oberbergkirchen, Germany ${ }_{2}^{2}$ Psychiatric Hospital, Ludwig Maximilian University, Munich, Germany ${ }^{3}$ LVR-Klinikum Essen, Universität Duisburg-Essen, Suchtforschungsgruppe an der Klinik für Psychiatrie und Psychotherapie, Essen, Germany

${ }^{4}$ Praxiszentrum Kaiserdamm, Berlin, Germany

${ }^{5}$ PiT - Praxis im Tal. Munich, Germany

${ }^{6}$ Privatklinik Meiringen, Willigen, Meiringen, Switzerland

\section{References}

1 Amato L, Minozzi S, Davoli M et al. Psychosocial combined with agonist maintenance treatments [Intervention Review]. Cochrane Database of Systematic Reviews 2011; (Issue 10)

2 Kamien JB, Branstetter SA, Amass L. Buprenorphine-naloxone versus methadone maintenance therapy: a randomized double blind trial with opioid-dependent patients. Heroin Addict Relat Clin Probl 2008; 10: $5-18$

3 Hser Y-I, Hoffmann V, Grella CF et al. A 33-year follow-up of narcotics addicts. Arch Gen Psychiatry 2001; 58: 503-508

4 Soyka M, Traeder A, Klotsche J et al. Criminal behavior in opioiddependent patients before and during maintenance therapy: 6-year follow-up of a nationally representative cohort sample. J Forensic Sci 2012, doi:10.1111/j.1556-4029.2012.02234.x

5 Federal Ministry of Health. Drug and Addiction Report 2001

6 NICE. NICE guidance. (National Institute for Health and Clinical Excellence) 2012. From http://guidance.nice.org.uk/

7 APA. American Psychological Association, 2012. From http://www. apa.org/

8 Kleber HD, Weiss RD, Anton RF et al. Treatment of patients with substance use disorders, second edition. American Psychiatic Association. Am J Psychiatry 2006; 163: 5-82

9 Soyka M, Kranzler HR, van den Brink $W$ et al. The World Federation of Societies of Biological Psychiatry (WFSBP) Guidelines for the Biological Treatment of Substance Use and Related Disorders. Part 2: Opioid dependence. World J Biol Psychiatry 2011; 12: 160-187

10 Wittchen $\mathrm{H}-U$, Apelt SM, Bühringer $\mathrm{G}$ et al. Buprenorphine and methadone in the treatment of opioid dependence: methods and design of the COBRA study. Int J Methods Psychiatric Res 2005; 14: 14-28

11 Federal Institute for Drugs and Medical Devices. 2011. From www. bfarm.de

12 Comer SD, Walker EA, Collins EC. Buprenorphine/naloxone reduces the reinforcing and subjective effects of heroin in heroin-dependent volunteers. Psychpharmacology 2005; 181: 664-675

13 Connock M, Juarez-Garcia A, Jowett $S$ et al. Methadone and buprenorphine for the management of opioid dependence: a systematic review and economic evaluation. Health Technol Assess 2007; 11: 1-171

14 Mattic R, Ali R, White J et al. Buprenorphine versus methadone maintenance therapy: a randomized double-blind trial with 405 opioiddependent patients. Addiction 2003; 98: 441-452

15 Johanson C-E, Arfken CL, di Menza $S$ et al. Diversion and abuse of buprenorphine: Findings from national surveys. Drug and Alcohol Dependence 2012; 120: 190-195

16 Stroller KB, Bigelow GE, Walsh SL et al. EC. Effects of buprenorphine/ naloxone in opioid-dependent humans. Psychopharmacology 2001; 154: 230-242

17 Bell J, Byron G, Gibson A et al. A pilot study of buprenorphine-naloxone combination tablet (Suboxone ${ }^{\circledR}$ ). Drug and Alcohol Review 2004; 23 : 311-317

18 Johnson RE, McCagh JC. Buprenorphine and naloxone for heroin dependence. Current Psychiatry Reports 2000; 2: 519-526

19 Fudala PJ, Yu E, Macfadden $W$ et al. Effects of buprenorphine and naloxone in morphine-stabilized opioid addicts. Drug and Alcohol Dependence 1998; 50: 1-8 
20 Chian CN, Hawks RL. Pharmacokinetics of the combination tablet of buprenorphine and naloxone. Drug and Alcohol Dependence 2003; 70: $39-47$

21 Mendelson J, Jones RT. Clinical and pharmacological evaluation of buprenorphine and naloxone combination: why the $4: 1$ ratio for treatment? Drug and Alcohol Dependence 2003; 70: 29-37

22 Fudala PJ, Williford WO, Collins J et al. Office-based treatment of opiate addiction with a sublingual-tablet formulation of buprenorphine and naloxone. New Engl J Med 2003; 349: 949-958

23 Guy W. Clinical Global Impressions. In: ECDEU Assessment Manual for Psychopharmacology, revised. Rockville, MD: National Institute of Mental Health; 1976; 218-222

24 Handelsmann L, Cochrane KJ, Aronson MJ et al. Two new rating scales for opiate withdrawal. The American Journal of Drug and Alcohol Abuse 1987; 3: 293-308

25 Ware JE, Scherbourne CD. The MOS 36-Item Short-Form Health Survey (SF-36). Medical Care 1992; 30: 473-483

26 Derogatis LR. SCL-90-R. Self-Report Symptom Inventory. In: Scalarum CI. Internationale Skalen für Psychiatrie. Weinheim: Beltz; 1986

27 Franke GH. Die Symptom-Checkliste von Derogatis - Deutsche Version - (SCL-90-R). Manual. Göttingen: Beltz Test GmbH; 1995

28 Stata Corp. Stata Statistical Software: Release 9. College Station, TX: StataCorp LP; 2005

29 Maintenance and Support Services Organisation (MSSO). MedDRA: Medical Dictionary for Regulatory Activities - Release 11.1. International Federation of Pharmaceutical Manufacturers and Associations (IFPMA), 2008
30 Wittchen $H-U$, Apelt SM, Soyka $M$ et al. Feasibility and outcome of substitution treatment of heroin-dependent patients in specialized substitution centers and primary care facilities in Germany: A naturalistic study in 2694 patients. Drug and Alcohol Dependence 2008; 95: 245-257

31 Schwartz RP, Kelly SM, O'Grady KE et al. Randomized trial of standard methadone treatment compared to initiating methadone without counselling: 12-month findings. Addiction 2012; 107: 943-952

32 Curcio F, Franco T, Topa $M$ et al. Buprenorphine/naloxone vs. methadone in opioid dependence: a longitudinal survey. European Review for Medical and Pharmacological Sciences 2011; 871-874

33 Carrieri MP, Amass L, Lucas GM et al. Buprenorphine use: the international experience. Clin Infect Dis 2006; 43 (Suppl 4): 197-215

34 German Federal Ministry of Justice. Verordnung über das Verschreiben, die Abgabe und den Nachweis des Verbleibs von Betäubungsmitteln (Betäubungsmittel- Verschreibungsverordnung - BtMVV), 1998 http://www.gesetze-im-internet.de/btmvv_1998/index.html

35 Lieb M, Wittchen H-U, Palm $U$ et al. Psychiatric comorbidity in substitution treatment of opioid-dependent patients in primary care: Prevalence and impact on clinical features. Heroin Addiction \& Related Clinical Problems 2012; 12: 5-16 